\title{
A system for remote orthopedics rehabilitation
}

\author{
David Tacconi, Roberto Tomasi \\ CoRehab \\ Trento, Italy \\ david@corehab.com; roberto@corehab.com
}

\author{
Cristina Costa, Oscar Mayora \\ UBiNT, CREATE-NET \\ Trento, Italy \\ cristina.costa@create-net.org; \\ oscar.mayora@create-net.org
}

\begin{abstract}
In this paper we present a system prototype for supporting the orthopedics rehabilitation process at the hospital and at home. In the system, the patient gets immediate feedback on the executed exercises controlling a gaming interface, while the system collects data about the therapy progress. Physiotherapists are able to remotely monitor the patient activity, and to adjust therapy according to the data available and patient's capacity. During the conference, authors will demonstrate functions and the concept behind the system, and will exemplify the different scenarios of use of this innovative tool, allowing the conference participants to wear the sensors and experiment the system in first hand. The system is now at a prototype stage.
\end{abstract}

Keywords-orthopedic rehabilitation, remote therapy, orthopedics rehabilitaiton

\section{INTRODUCTION AND MOTIVATION}

With the recent diffusion of affordable and accessible motion capture technologies introduced by gaming platforms (i.e. Nintendo Wii, MS Kinect, Sony Playstation Move), we have seen an increasing interest on their usage in physical rehabilitation both within the clinic and home [1][3]. Up to now, research has mainly addressed, with positive results, the usage of such game based tools for the physical rehabilitation of patients with neurological damages following injury or disease to the nervous system (such as stroke, spinal cord injury (SCI), or traumatic brain injury (TBI)) [2]. In such cases, the rehabilitation main goal is the recovery of motor function through intense skillful practice, e.g. with exercises that improve patients' standing balance, strength, coordination and activity tolerance, or occupational therapies.

When it comes to orthopedics rehabilitation, however, main requirements change. There is a shift from the need to recover from a centralized brain injury, to the need of recuperate from a peripheral damage; a more precise monitoring of the movement is needed, in order to exercise the right muscles, avoiding compensation patterns or movements that increase the risk of injury: overworking or straining of the injured part during this period can compromise the success of a chirurgic intervention itself.

In orthopedics rehabilitation, the work done at the hospital is complemented, at different degrees depending on the injury, by a home program of exercises. The patients are taught at the hospital on how to perform correctly the exercises, but when alone at home, they must be able to execute the program without support. The success of a therapy depends on continuing the prescribed exercises once the patient is at home: exercises are often repetitive and sometimes painful, therefore motivation can be an issue, especially in the latest rehabilitation phases, when the main physical functions are recovered and the patient can get back to normal life. Therapist monitors the advancement of the therapy during the periodical checks, during which they may also check if the patients has found difficulties in executing the home program.

In this paper we present a prototype system, specifically designed to support the physical rehabilitation of orthopedics patients, both locally (at the hospital) and remotely (at home). The prototype is an integrated system that includes motion capture technologies, game interfaces and remote motoring, which is able to support both the patient and the physiotherapist during the whole rehabilitation process, starting from the hospital and following the patient at home.

\section{SYTEM DESCRIPTION}

The prototype is composed by a gaming interface, a backend system and a Web interface (Fig. 1). The interface was designed to be friendly and engaging for the patients while supporting them in their rehabilitation exercises. In the meanwhile the system allows the therapist to follow the patients' progress remotely and keep track of their day by day

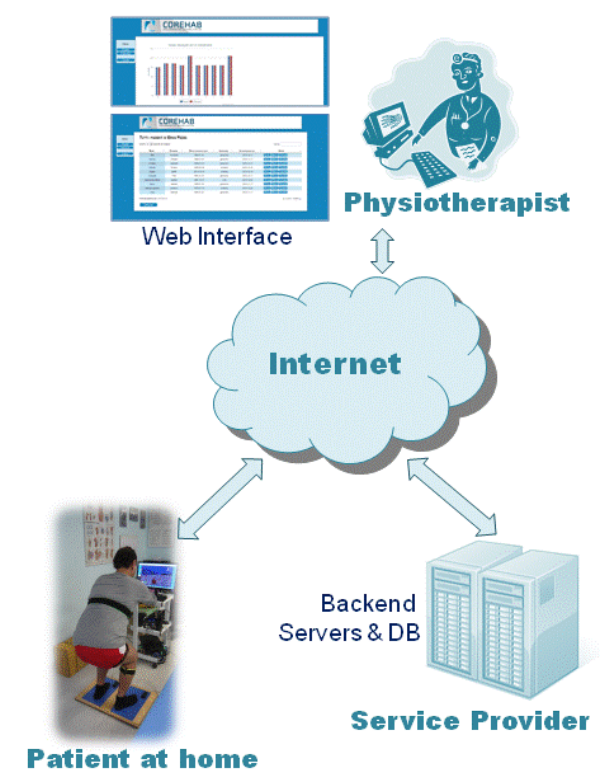

Figure 1 - Prototype architecture 
progress, highlighting possible points of problems.

\section{A. Motion tracking hardware - Data acquisition system}

We use a set of five minimal intrusive wearable high precision sensors with 3D accelerometers, gyroscopes and magnetometers, connected with Bluetooth and positioned on the body with elastic stripes. Sensors are supported in measurements by a board with embedded pressure sensors. Sensors monitors the correctness of the performed exercise in terms of the parameters relevant to the orthopedics rehabilitation therapy, such as joint angles, angles of the limbs, distribution of weight, balance, etc.

\section{B. Patient interface - Games}

The patient interface has been designed considering both rehabilitation and patients' requirements. The patient is guided in his exercises by a set of platform games, controlled by the wearable sensors and the board. Game mechanics reflects the rehabilitation exercise goals: for mobility exercises, for example, the associated exercises gradually propose the patient with movements with increasing amplitude, while continuing monitoring the progress in order to avoid overwork (Fig. 2b). Games give the right weight and pace to the different phases of an exercise, control movements in order to avoid dangerous movements, give feedback on the correctness of the exercise, and keep the attention of the patient adapting the game to patient's abilities. When an exercise is correctly executed, the patient is able to collect rewards. In the latest

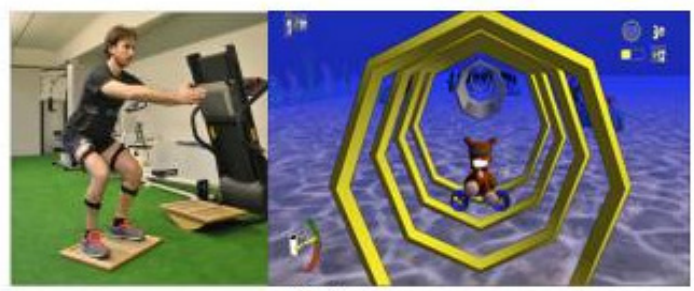

(a)

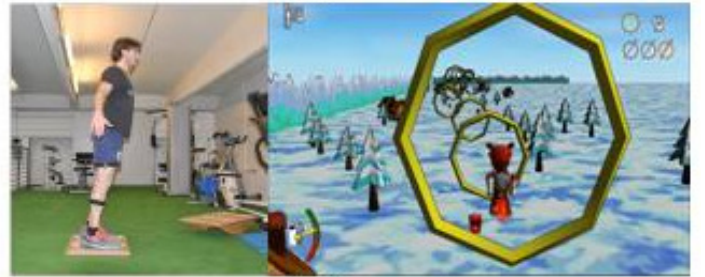

(b)

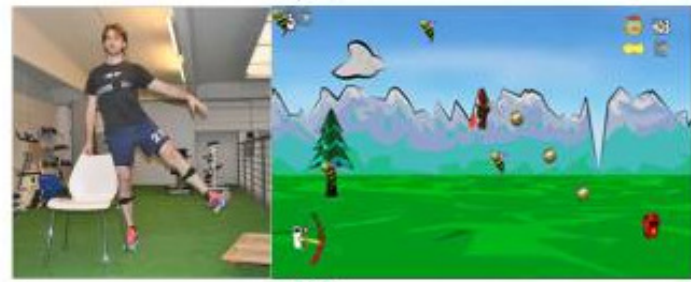

(c)

Figure 2 - Exercises and Game Interfaces: (a) Squat; (b) Weight shifting; (c) Hip abduction prototype, the developed games allow the execution of twenty exercises, tailored for different phases of knee injury and back pain rehabilitation, and include both isometric and mobility exercises. The exercises are organized in programs that can be personalized for each patient.

The range of this parameters within which the exercise is considered correct during execution is set in advance by the physiotherapist through a Web interface. Feedbacks to the user are given both in terms of rewards collected and in terms of the correctness of the exercise.

The system gives the patient an active role in the therapy, acting on the patient motivation and supporting him or her in performing the assigned exercises correctly and independently, thus improving the effectiveness of the therapy.

\section{Backend system and Terapist interface}

Motion data is collected by a centralized database that keeps track of the progress of all patients, and maintains the information on the programs. The backend system analyzes the data and provides it asynchronously to the physiotherapist. A web application allows the therapist to set up the rehabilitation program for the patient (exercises type, number of series and repetitions, parameters in terms of angles and balance percentage) and to monitor how well the patient is performing. Therapists can remotely access to the session data recorded and adjust the program if needed.

The system provides therapists with a wider and more comprehensive perspective on the therapy progress, maintaining historical data and supporting different visualizations of rehabilitation data.

\section{DEMO PRESENTATION}

The prototype that will be presented is currently in use for testing in a local hospital where patients with knee injuries and back pain are able to use the system during their regular therapy session. The prototype presented at the conference will be available to the attendees who will be able to wear the sensors and try the various exercise programs. It will be also possible to explore the collected data through the therapist Web interface.

\section{REFERENCES}

[1] Lange, B., Flynn, S. and Rizzo, A. Initial usability assessment of off-theshelf video game consoles for clinical game-based motor rehabilitation. Physical Therapy Reviews, V.14 N.5 pp.355-363, 2009.

[2] Lewis GN, Rosie JA. Virtual reality games for movement rehabilitation in neurological conditions: how do we meet the needs and expectations of the users? Disability Rehabilitation. V.34 N.22 pp.1880-1886, 2012

[3] Lange, B.S., Koenig, S., Chang, C., McConnell, E., Suma, E., Bolas, M. \& Rizzo. A. Designing Informed Game-Based Rehabilitation Tasks Leveraging Advances in Virtual Reality. Disability and Rehabilitation, V.34 N.22 pp.1863-1870, 2012. 